\title{
Recurrent Waldenstrom Macroglobulinemia
}

National Cancer Institute

\section{Source}

National Cancer Institute. Recurrent Waldenstrom Macroglobulinemia. NCI Thesaurus.

Code C127840.

The reemergence of Waldenstrom macrog lobulinemia after a period of remission. 